\title{
E. Schlußbemerkungen und Ausblick
}

Am Anfang war Hinkmar. Am Ende dieser Arbeit jedoch sucht man vergeblich nach ihm. Hier stehen vielmehr unzählige vielschichtige Einzelergebnisse aus isolierten Erinnerungsbildern, die in viele Richtungen weisen, an zahlreiche Orte, in mehrere Jahrhunderte, zu verschiedenen Personen: Reims, Trier, Echternach, Mettlach, Karlmann, Tilpin, Rigobert, Abel, Liutwin, Basin, Irmina, Egbert, Thiofrid, Theoderich, um nur einige zu nennen. Die heterogenen Einzelbilder lassen sich zu keinem Gesamtbild aneinanderfügen, sondern stehen in den meisten Fällen allein; sie sind unabhängig voneinander. Die jeweiligen Ergebnisse lassen sich über die entsprechenden Summen der drei Hauptkapitel erschließen. Welche Konsequenzen haben die Ergebnisse dieser Arbeit für die historische Erkenntnis im allgemeinen? Methodisch betrachtet läßt sich die hier deutlich gewordene Kontingenz historischer Erinnerungsbilder nicht auf diesen Einzelfall beschränken.

Am Anfang war also Hinkmar, doch am Ende ist kaum etwas geblieben von den Bildern, die er in seiner Denkschrift über das 8. Jahrhundert in Reims und Trier überliefert. Zwar erwies sich seine Sichtweise als wirkmächtig bis in die jüngste Zeit. Doch hat sich im Verlauf dieser Untersuchung gezeigt, daß Hinkmar nicht abbildete, was einmal geschah, sondern das, was seiner Meinung nach einmal so geschehen sein sollte. Dies unterstreicht die Situativität und Konstruktivität einer jeden Quelle. Historiker allerdings woben daraus, verbunden mit anderen Quellen, einen Teppich, der fest genug schien, die Geschichte der Kirche von Reims und Trier im früheren Mittelalter zu tragen, fest genug auch, um ihn mit weiteren Erkenntnissen aus anderen Bereichen zu belasten. Sie verließen sich auf das, was sie vorfanden, was überzeugte, und versuchten, die wenigen kleinen, abweichenden Spuren noch mit in das Geflecht einzupassen. Sie schufen zwar ein anschauliches Gesamtbild, ließen aber eine weitere Dimension außer acht: die der Zeit, in deren Fluß die Quellen unentwegt neue Erinnerungsbilder eines Gegenstandes entwerfen, von denen keines dem anderen gleicht, bis oft der ursprüngliche Gegenstand selbst einmal nicht mehr auszumachen ist oder in Vergessenheit gerät. Folgt man also dem Fluß dieser kontingenten Bilder in chronologischen Schritten ausgehend von seiner Quelle bzw. löst man einen der tragenden Fäden aus dem Teppich und studiert die Kräfte, die ihn formten, so zerreißt das ganze Geflecht.

An dieser Stelle soll exemplarisch für das gesamte betrachtete Material nur ein solcher Faden herausgegriffen werden, der alle drei Untersuchungskapitel deutlich durchzieht, um zu zeigen, wie er ganz allmählich, dynamisch und 
unkontrollierbar gesponnen wurde. Es handelt sich um Milo, von dem wir im Laufe der Arbeit vielfältige Erinnerungsbilder gewonnen haben. Wie gestaltet sich der Fluß dieser Bilder, wenn man ihn in seiner Länge überblickt? Betrachten wir dessen Genese:

$\mathrm{Zu}$ Beginn stand ein Adliger oder niederer Kleriker namens Milo im früheren 8. Jahrhundert, der die Trierer Bischofspfalz bzw. die bischöfliche Wirtschaft, das dortige episcopium, verwaltete, das er vielleicht von Karl Martell übertragen bekommen hatte. Einigen Klerikern wie Bonifatius dürfte das nicht genehm gewesen sein, weshalb er beim Papst gegen ihn polemisierte, den Schaden für die Kirche betonte. Doch so viel Schaden scheint Milo nicht verursacht zu haben. Denn er sandte die amtierenden Trierer Bischöfe zusätzlich als Äbte nach Mettlach, ins Kloster seiner Familie. Milo gehörte einer bedeutenden Sippe an und stand in unmittelbarer Königsnähe. Er starb etwa in der Mitte der 750er Jahre und wurde in Mettlach bestattet, wo man sich seiner noch über 300 Jahre nach seinem Tod positiv erinnerte. Ab der Mitte des 9. Jahrhunderts legte sich ein neues Bild darüber, das einen anderen Milo zeigte und den beschriebenen verdeckte: Hinkmar von Reims verband Milo nun auch mit dem Reimser episcopium. Ob ihm dafür heute verlorene Quellen zur Verfügung standen, läßt sich nicht mehr ermitteln. Doch wird aus dem oeconomus nun ein Okkupant beider Bistümer. Denn Hinkmar las episcopium nicht mehr im (wohl älteren) Sinne von ,Wirtschaftsgebäuden', sondern als ,Bistum'. Er verstand Milo deshalb als unrechtmäßigen Bischof, obwohl dieser ein solches Amt niemals bekleidet hatte, und interpretierte ihn als Kleriker nach der Tonsur, aber Laien nach seinem Verhalten und als einen Entfremder von Kirchengut. Ein negatives Bild war geschaffen, das Hinkmar bei der Argumentation zum verlorenen Reimser Kirchenbesitz seiner eigenen Zeit einsetzte. Hinkmar ließ in seinen Texten Milos Herrschaft auf mehr als vierzig Jahre anwachsen, worüber Bischöfe wie der Reimser Abel in Vergessenheit gerieten.

Dieses neue Erinnerungsbild erwies sich als wirkmächtig, wurde langsam um zusätzliche Facetten ergänzt, bis es im 10. Jahrhundert einen festen Platz in Flodoards „Reimser Kirchengeschichte“ erhielt. Über diese sowie gemeinsam mit weiteren Reimser Texten gelangte es in den Trierer Raum und kollidierte mit dem dortigen positiven Milobild, das man im Kloster Mettlach pflegte. Infolge formten sich neue Erinnerungsbilder, indem sich die bestehenden einander annäherten und die Vergangenheit neu aushandelten: Der eigentlich gute Milo mußte sich also langsam zu einem schlechten Milo gewandelt haben. Deshalb wurden seine positiven und negativen Attribute nun in Form rein positiver Bilder als ,qualitative Inversion' auf die Gestalt seines vermeintlichen Vaters Liutwin projiziert. Auch in der Trierer Überlieferung wurde Milo nun zum unrechtmäßigen Doppelbischof. Seine vorgeblich lange Amtszeit unterdrückte die Bilder und Namen der einmal tatsächlich amtierenden Trierer Bischöfe. Milos Niedertracht im Doppelamt allerdings bedrohte die Trierer Pri- 
matsansprüche im späten 10. Jahrhundert über die Gallia Belgica und Germania. Liutwin ersetzte deshalb das getrübte Bild Milos als strahlendes Gegenbild. Als fürsorglicher Bischof wirkte er nun in drei Bistümern: Reims, Trier und Laon. In Mettlach entstand damals der Kult eines hl. Liutwin, begleitet vom Neubau einer für sein Grab vorgesehenen Kirche, der Translation seiner Reliquien von Trier nach Mettlach sowie einer ersten Liutwin-Vita. In Trier wollte man dagegen Milos Mettlacher Memoria verdrängen. In der Mitte des 11. Jahrhunderts behauptete man im Erzstift sogar, der Bischof sei seinem Lebensstil angemessen auf der Eberjagd gestorben und im Weiler Ehrang begraben worden. In Mettlach hingegen hielt man an Milo fest, ließ ihn als positives Bild gleichwertig neben dem des Vaters bestehen. Beider Bilder emanzipierten sich schließlich voneinander und fanden sich bald auch in Trier wieder.

Diese Einzelbilder heben nicht nur das alte Gesamtbild der Forschung auf, sie lösen auch deren Konstrukte ab, die diese als Thesen zum besseren Verständnis einzelner Phänomene aufstellte. So muß die genaue Form der Opposition gegen Bonifatius im Frankenreich überdacht werden. Der Parallelismus von amtierenden Reimser und Trierer Bischöfen neben Milo braucht nicht mehr mit zusätzlichen Kloster-, Abt- oder Chorbischöfen erklärt zu werden.

Dieses Beispiel zeigt stellvertretend für alle weiteren Einzelergebnisse die Kontingenz der betrachteten Reimser und Trierer Geschichtsbilder, die jedoch keineswegs hierauf beschränkt sein dürfte. Gerade Themen mit dünner Quellenüberlieferung laufen Gefahr, diese Kontingenz zu überdecken, weil sich der Wandel der Erinnerungsbilder auf den ersten Blick nur schwer erschließt.

Als methodische Konsequenzen für die historische Analyse solcher Bilder ergeben sich die folgenden Überlegungen:

1. Die für diese Arbeit gewählte Vorgehensweise, die Quellen zu einer Thematik ausgehend von deren Ursprung chronologisch fortschreitend, kontextualisierend und kleinteilig zu prüfen, hat sich im Verlauf der Untersuchungen als unabdingbar bestätigt. Letztlich muß man jede Geschichtsquelle als eigenständiges, einzigartiges, einmaliges Bild verstehen. Akzeptiert man das, so lassen sich einzelne Bildstufen der Quellen klar und analytisch voneinander trennen, isolieren und Verformungen nachzeichnen. Allein auf diese Weise vermag man, die ungeheure Kontingenz der Einzelbilder zu bewältigen und sie nicht monoperspektivisch, sondern multiperspektivisch zu strukturieren. Damit läßt sich der Situativität jeder einzelnen Überlieferungsebene Rechnung tragen. Auch Vermischungen verschiedener Diskurse sind analytisch einfacher zu erkennen und auseinanderzuhalten. Das Instrumentarium bietet eine größere Sicherheit beim Herausfiltern auf den ersten Blick unauffälliger Details, die sonst in der Bilderflut untergingen. Gerade solche Details können Reste alter Bilder darstellen, die andernfalls oft bereits verformt oder von neuen, wirkmächtigeren überdeckt worden sind. Derartige Details bieten beispielsweise die Zeugenlisten der Irmina-Urkunden, die noch etwas über das ursprüngliche 
Aussehen eines „Ur-Testaments“ verraten. Man darf es bei diesem chronologischen Vorgehen aber nicht dabei belassen, allen vorgegebenen Datierungen zu vertrauen. Vielmehr ist eine Gegenprobe erforderlich, ob sich die Quelle in ihren vermeintlichen Kontext tatsächlich einfügt oder an ganz anderer Stelle besser paßt.

Die gerade beschriebene Arbeitstechnik ist eine kartographische oder archäologische. Es geht zunächst darum, die Quellen, die die Bilder überliefern, genauestens zu vermessen und einem engmaschigen dreidimensionalen Raster zuzuweisen. Der alte Quellenteppich muß dazu Schicht um Schicht aufgetrennt und aufgezeichnet werden, um zunächst eine relative Chronologie anzulegen. Schließlich können vorhandene, sicher bestimmbare Zeugnisse es ermöglichen, eine absolute Chronologie zu erstellen. Jedes Fundstück muß nach seiner eigentlichen Herkunft bestimmt und kontextualisiert werden. Gehört es also tatsächlich in die Schicht, in der es angetroffen wird? Das systematisierende Raster dient dazu, das vorhandene Material detailliert auszubreiten, zu sortieren und anzuordnen. Dieses äußere Gerüst kann durch eine sich vielleicht bereits ergebende Struktur zu ersten Ergebnissen und Erkenntnissen führen. Man erhält damit Ebene für Ebene zahlreiche, in gewisser Weise objektiv beschreibbare Planums, die die Fundlage dokumentieren, bevor man mit der Interpretation beginnen kann. Mit anderen Worten: Es geht hier um den Versuch, den physischen Verlauf des Flußbettes zu rekonstruieren, ohne es schon mit Wasser bzw. Inhalt zu füllen. Man hat also zunächst alle noch verfügbaren Quellen zur genaueren Untersuchung der Bildgenese eines Themas in einem solchen Raster anzuordnen.

2. Nachdem die Quellen als Überlieferungsträger der Bilder vermessen worden sind, sind nun die Bilder selbst, ihre Inhalte und Einzelfacetten, genauestens zu betrachten. Dabei gilt es, sich am Raster bzw. dem Flußlauf zu orientieren. Die Abfolgen der Bilder zum gewählten Thema müssen präzise nachvollzogen und dabei zu beobachtende Veränderungen und Verformungen zunächst minutiös festgehalten werden. Lassen sich Bilder und Veränderungen bereits aus dem jeweiligen Kontext verstehen? Welche Rückschlüsse bieten sie darüber? Übernehmen die Bilder vorausgehende Bilder oder nur einige ihrer Elemente? Verändern sich diese? Kommen neue von ganz anderer Seite hinzu, werden auch sie verändert? Woher genau sind sie eingeflossen? In dieser Arbeit hat sich gezeigt, daß vor allem Personen - wie eben auch Milo - als Projektionsfläche solcher Bilder dienen, seien sie nun real oder fiktiv. So transportieren Personen ihnen zugeschriebene Attribute oder Funktionen, die sich unablässig weiter verändern. Gleichermaßen gilt dies aber auch für Orte, besondere bildhaft begründbare Rechtskonstruktionen oder einzelne Begrifflichkeiten, die im Laufe mehrerer Jahre anders verstanden werden können. Orientiert man sich also zunächst an diesen besonders charakteristischen Gruppen, sind erste $\mathrm{Zu}$ - 
gänge zum Verständnis der Einzelbilder und ihres Wandels möglich, über die sich gegebenenfalls weitere Facetten erschließen.

3. Der Fluß der Bilder ist unterschiedlichen Verformungsfaktoren unterworfen, die auf ihn einwirken und die es deshalb grundsätzlich zu berücksichtigen gilt. Erst über sie erschließt sich die Form der jeweiligen Einzelbilder im ausgebreiteten Raster. Der dabei primär zu beachtende Faktor ist das individuelle Gedächtnis. Bilder werden in einer jeweiligen Situation immer wieder neu erinnert und somit immer wieder neu geschaffen. Warum erinnert also der Verfasser eines Erinnerungszeugnisses gerade jetzt, was er erinnert? Welche unbewußten inneren Elemente des je eigenen Gedächtnisses, des Gewußten und der eigenen Prägung sind an der Konstruktion seines Erinnerungsbildes beteiligt? Gibt es unbewußte äußere Schlüsselreize, die seine Erinnerung leiten und beeinflussen? Handelt es sich dabei vielleicht um einen Text, den der Verfasser gerade studiert hat, womöglich um ein Objekt, das er sieht, eine Naturerscheinung, eine Lesung im morgendlichen Gebet? Geben gar bekannte Legenden einen Impuls oder Namen, die zu Analogiebildungen anregen? Wesentlich ist also, gründlich nach diesen inneren und äußeren Reizen für das individuelle Gedächtnis im Umfeld des Erinnerungsbildes zu suchen, um klären zu können, wie weit die Entstehung eines Bildes davon beeinflußt wird.

Neben solchen unbewußten, individuellen Faktoren, die Erinnerungsbilder ganz entscheidend prägen, existieren aber auch bewußte äußere Faktoren, die dabei mitwirken: willentliche Konstruktionen nämlich, bis hin zu Fälschungen. Auf diese Weise lassen sich Erinnerungsbilder gezielt entwerfen. Wir haben dies an den Mettlacher Diplomen und den Echternacher Irmina-Urkunden verfolgt. Dabei ist bestätigt worden, daß grundsätzlich immer authentisch erscheinende Vorlagen in diese Bilder miteinbezogen werden, die im unmittelbaren Umfeld greifbar sind. Man muß also im Kontext eines Bildes prüfen, ob es sich um eine bewußte Konstruktion handelt; ob authentische Vorlagen an Ort und Stelle vorhanden sind. Welche älteren Texte liegen vor? Über welche Handschriften verfügt man? Besteht Kontakt zu benachbarten Wissenszentren? Gibt es einflußreiche Heiligenkulte, die sich gerade verbreiten? Sind ältere Urkunden bekannt, die man interpolieren, verunechten oder nach denen man fälschen kann? Bieten sich alte Gräber, Sarkophage, Architektur dazu an, diese umzudeuten? Schließlich: Durchmischen sich unbewußte und bewußte Phänomene beim Entstehen der Geschichtsbilder?

4. Die Frage ist endlich, wie genau die im stetigen Fluß befindlichen, durch unbewußte und bewußte Faktoren bestimmten Bilder sich untereinander und nacheinander beeinflußten und verformten. Man muß dazu zwischen den einzelnen Objekten des dreidimensionalen Rasters gleichsam Verbindungsfäden anbringen, um dies zu visualisieren. Allerdings ergeben diese keinen Teppich mehr, sondern ein dreidimensionales Netz. Gerade der dritten Dimension, der Zeit, kommt eine kaum zu überschätzende Bedeutung zu. Nur sie trägt der 
Kontingenz der Bilder Rechnung, nur über sie lassen sich die zahllosen Verformungsprozesse genauer kartographieren.

Neben den Verformungen der menschlichen Erinnerung und den bewußten Konstruktionen wirkt auch die „Macht“ des Diskurses auf die Bilder ein. Es ist also ebenso erforderlich zu prüfen, welche politischen, sozialen etc. Phänomene es jeweils ermöglichen, daß einige Bilder eine höhere oder geringere Wirkmächtigkeit entfalten als andere. Der dynamische, von all diesen Faktoren bestimmte, unvorhersehbare Prozeß der Erinnerungsbilder findet in der dritten Dimension eine diachrone Ebene des Bildaustauschs, die dem „kollektiven Gedächtnis" entspricht.

Die Kontingenz von Geschichts- bzw. Erinnerungsbildern beginnt mit dem Ereignis, mit der Verformung im individuellen Gedächtnis oder durch eine bewußte Konstruktion. Beide gehen dann unmittelbar ins „kollektive Gedächtnis“ über, d.h. sowohl ins „kommunikative Gedächtnis“ einer Generation wie in das darüber hinaus wirkende „kulturelle Gedächtnis“. Zwischen den einzelnen beteiligten individuellen Gedächtnissen beginnen im kollektiven Gedächtnis umgehend die ersten Aushandlungsprozesse über die jeweiligen Bilder. Das „kollektive Gedächtnis“ ist wiederum von der „Macht“ des Diskurses beeinflußt.

Man muß sich die unendliche Kontingenz der Bilder also überall und jederzeit vergegenwärtigen: bei jedem noch so unbedeutend erscheinenden Bild, aus dem sich wiederum größere zusammensetzen, ist sie wirksam. Deshalb ist es dringend geboten, eine Quelle nicht mehr als statischen Baustein für ein großes zu erschließendes Gesamtbild anzusehen, der nur mit weiteren solcher Bausteine verbunden werden müßte. Vielmehr ist jede Quelle, jedes Bild das Produkt eines stets hochdynamischen Prozesses, der immer mitgedacht werden muß. Der Historiker verfügt also nur über kurze Momentaufnahmen des Erinnerungsflusses und kann häufig kaum mehr sagen, was noch einige Wochen, Tage, Stunden, Minuten zuvor und danach oder gar zur gleichen Zeit an einer etwas anderen Stelle gedacht oder erinnert worden ist. Um zu rekonstruieren, was dazwischen liegen könnte, benötigt der Historiker das dreidimensionale Raster. Spinnt man also zwischen all den darin befindlichen Bildern die entsprechend nachweisbaren Fäden, zeigt sich mit gewisser Wahrscheinlichkeit, ob einige davon zu einem einstigen Ereignis zurückführen, das seine Wirkung entfaltet hat, oder ob solche Bilder über Seitenlinien des Flusses in diesen dynamischen Prozeß hineinströmen und so auf ihn einwirken.

Damit müßte nachvollziehbar werden, warum eine Quelle, die sich einige Jahre nach einem Ereignis an einer entsprechenden Stelle des Rasters befindet, ohne weitere erhaltene Bilder im Raster kaum sichere Aussagen über das beschriebene Ereignis noch ihren eigenen inhaltlichen Wert ermöglicht. Vielmehr zwingt die anzunehmende Kontingenz der Bilder, die Quelle als verläßliches Zeugnis zunächst abzulehnen und mit größter Vorsicht zu behandeln. 
Einige der Bilder sind stärker und wirkmächtiger als andere. So vermag das anfangs beschriebene Bild Hinkmars die ihm vorausgehenden fast völlig $\mathrm{zu}$ verdecken, wie ein Wehr oder eine Schleuse, die den dynamischen Bilderfluß aufstaut, den direkten Zugang zu seinem Oberlauf versperrt und den Abfluß des Wassers kurzfristig dosiert. Solche Hindernisse bilden Kulminationspunkte, an denen sich Bilder besonders verdichten und damit auf die Fließgeschwindigkeit einwirken können. Nur die wenigen Steinchen, die herübergespült werden, bieten dann noch Auskunft darüber, was davor lag. Bedenkt man aber das Hindernis, das sie überwunden haben, ist ihr Wert um so höher einzuschätzen als der der Bilder, die mit größerer Leichtigkeit nach dem Hindernis entstehen. Stoßen wir in unserem Raster also auf eine Quelle mit einem facettenreichen Bild, das zahlreiche Fäden mit weiteren sprudelnden Bildern verbindet, vor dem aber eigenartigerweise kaum solche Bilder zu finden sind, müssen wir mit einem derartigen Befund rechnen. Er sollte immer Verdacht erregen. Das Raster kann jedoch dabei helfen, die Genese dieses wirkmächtigen Bildes zu erklären.

5. Um die Kontingenz von Geschichts- und Erinnerungsbildern anhand einer historischen Quellen- und Gedächtniskritik untersuchen zu können, ist also mehr als nur eine Gedächtnisgeschichte erforderlich, die sich mit der Bildrezeption beschäftigt. Vielmehr benötigen wir eine Archäologie eben dieser Bilder, die ein bestehendes monoperspektivisches Gesamtbild Schicht um Schicht abträgt, um es in all seiner Heterogenität und Multiperspektivität sichtbar zu machen. Damit geht zwangsläufig das ursprüngliche Gesamtbild verloren. Statt dessen aber wird der Bilderfluß im einzelnen dokumentiert und kartographiert. Die Ergebnisse eines jeden gewonnenen Planums können ganz neue Erkenntnisse für den Kontext bieten, in dem es steht, bis eben hinauf zur Quelle. Bei einer solchen Untersuchung wächst dann schrittweise ein auf den ersten Blick verwirrendes dreidimensionales Netz unzähliger Bündel, das auf den zweiten mit seiner erkennbar werdenden komplexeren Struktur ganz neue Einsichten ermöglicht.

Auf diese Weise kann der anfangs beschriebene Konstruktionskreislauf der Geschichtsbilder nachvollziehbar gemacht werden. Erst wenn man diese methodischen Konsequenzen berücksichtigt, wird sich zeigen, wie sicher das Faktengerüst tatsächlich trägt, mit dem man bislang gearbeitet hat, und was davon nur mißverstandener Teil eines sich wiederholenden, unendlichen Bilderflusses gewesen ist.

„Alle Wasser laufen ins Meer, doch wird das Meer nicht voller; an den Ort, dahin sie fließen, fließen sie immer wieder." (Prediger/Kohelet 1,7) 
